\title{
Trends in the epidemiology of urinary tract infections in pregnancy at a tertiary hospital in Johannesburg: Are contemporary treatment recommendations appropriate?
}

\begin{tabular}{|c|c|}
\hline \multicolumn{2}{|c|}{$\begin{array}{l}\text { Authors: } \\
\text { Trusha Nana }{ }^{1,2} \\
\text { Shastra Bhoora } \\
\text { Vindana Chibabhai }^{3,4,5}\end{array}$} \\
\hline \multicolumn{2}{|c|}{$\begin{array}{l}\text { Affiliations: } \\
{ }^{1} \text { Department of Clinical } \\
\text { Microbiology and Infectious } \\
\text { Diseases, University of } \\
\text { the Witwatersrand, } \\
\text { Johannesburg, South Africa }\end{array}$} \\
\hline \multicolumn{2}{|c|}{$\begin{array}{l}{ }^{2} \text { Microbiology Laboratory, } \\
\text { Charlotte Maxeke } \\
\text { Johannesburg Academic } \\
\text { Hospital, National Health } \\
\text { Laboratory Services, } \\
\text { Johannesburg, South Africa }\end{array}$} \\
\hline \multicolumn{2}{|c|}{$\begin{array}{l}{ }^{3} \text { Department of Obstetrics } \\
\text { and Gynaecology, University } \\
\text { of the Witwatersrand, } \\
\text { Johannesburg, South Africa }\end{array}$} \\
\hline \multicolumn{2}{|c|}{$\begin{array}{l}{ }^{4} \text { Department of Obstetrics } \\
\text { and Gynaecology, Charlotte } \\
\text { Maxeke Johannesburg } \\
\text { Academic Hospital, } \\
\text { South Africa }\end{array}$} \\
\hline \multicolumn{2}{|c|}{$\begin{array}{l}{ }^{5} \text { Gauteng Department of } \\
\text { Health, Johannesburg, } \\
\text { South Africa }\end{array}$} \\
\hline \multicolumn{2}{|c|}{$\begin{array}{l}{ }^{6} \text { Department of Obstetrics } \\
\text { and Gynaecology, Faculty of } \\
\text { Medicine, Baylor College, } \\
\text { Houston, United States } \\
\text { of America }\end{array}$} \\
\hline \multicolumn{2}{|c|}{$\begin{array}{l}\text { Corresponding author: } \\
\text { Trusha Nana, } \\
\text { trusha.nana@nhls.ac.za }\end{array}$} \\
\hline \multicolumn{2}{|c|}{$\begin{array}{l}\text { Dates: } \\
\text { Received: } 21 \text { Aug. } 2021 \\
\text { Accepted: } 19 \text { Oct. } 2021 \\
\text { Published: } 09 \text { Dec. } 2021\end{array}$} \\
\hline \multicolumn{2}{|l|}{ Read online: } \\
\hline 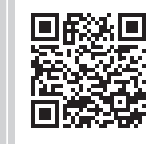 & $\begin{array}{l}\text { Scan this QR } \\
\text { code with your } \\
\text { smart phone or } \\
\text { mobile device } \\
\text { to read online. }\end{array}$ \\
\hline
\end{tabular}

Background: Urinary tract infections (UTIs) are common during pregnancy and are associated with maternal and foetal complications. Empiric antibiotic choices in pregnancy require consideration of efficacy and safety, resulting in limited oral options. With rapidly evolving antibiotic resistance, surveillance to guide empiric treatment recommendations is essential.

Methods: A retrospective analysis of urine culture isolates from the Charlotte Maxeke Johannesburg Academic Hospital (CMJAH) Obstetrics Department for 1 January 2015 to 31 December 2020 was performed.

Results: The top 3 pathogens were Escherichia coli, Enterococcus faecalis and Klebsiella pneumoniae. For E. coli susceptibility to cefuroxime declined ( $95 \%$ to $81 \%, p<0.0001)$. Similarly, the E. coli extended spectrum beta-lactamase rate increased from $5 \%$ to $10 \%(p=0.04)$. E. coli susceptibility to nitrofurantoin (93\%) and fosfomycin (96\%) remained high. In 2019, carbapenem-resistant K. pneumoniae emerged. Ampicillin susceptibility was high amongst the E. faecalis isolates. Amoxicillin-clavulanate demonstrated high levels of activity against the top 3 uropathogens.

Conclusion: The Essential Drug List recommended antibiotics for lower UTIs, nitrofurantoin and fosfomycin, are appropriate empiric options for E. coli, the most common uropathogen in the CMJAH obstetric population. The high rate of $E$. faecalis susceptibility to nitrofurantoin reported from other Gauteng tertiary obstetric patients, suggests that nitrofurantoin will provide adequate empiric cover for a large proportion of UTIs. However, the determination of the E. faecalis nitrofurantoin and fosfomycin susceptibility rates in the CMJAH obstetric population will provide useful data. Periodic surveillance at the various levels of antenatal care in different regions of South Africa and the determination of risk factors for infections with resistant uropathogens is needed.

Keywords: urinary tract infections; asymptomatic bacteriuria; pregnancy; antimicrobial resistance; surveillance; trends.

\section{Background}

Urinary tract infections (UTIs) are common during pregnancy. The hormonal and anatomical changes that occur during each trimester render pregnant women vulnerable to UTIs. In the first trimester, progesterone promotes smooth muscle relaxation and the ureters begin to dilate. Towards the mid-second trimester, the growing gravid uterus compresses the urogenital system, thus worsening hydronephrosis, urinary stasis and utero-vesical reflux. These collective adaptations favour asymptomatic and symptomatic UTIs. ${ }^{1}$ Asymptomatic bacteriuria (ASB), defined as the presence of $\geq 10^{5}$ colony forming units (CFUs) $/ \mathrm{mL}$ of a bacterial species in a voided urine specimen in the absence of UTI symptoms or signs, is most common. ${ }^{2}$ Asymptomatic bacteriuria occurs in $2 \%-15 \%$ of pregnancies and is associated with an increased risk of preterm labour. ${ }^{1,2,3,4}$ Screening for and treatment of ASB are recommended early in pregnancy. ${ }^{2,3}$ Treatment of ASB decreases the risk of pyelonephritis and may also reduce premature birth and very low

How to cite this article: Nana T, Bhoora S, Chibabhai V. Trends in the epidemiology of urinary tract infections in pregnancy at a tertiary hospital in Johannesburg: Are contemporary treatment recommendations appropriate? S Afr J Infect Dis. 2021;36(1), a328. https://doi. org/10.4102/sajid.v36i1.328

Copyright: ( 2021. The Authors. Licensee: AOSIS. This work is licensed under the Creative Commons Attribution License. 
birth weight deliveries. ${ }^{2}$ Symptomatic UTIs, cystitis and pyelonephritis complicate $1 \%-2 \%$ of pregnancies and are associated with several maternal and foetal complications. ${ }^{1,4}$

Current epidemiologic data are required to guide empiric antibiotic choices. Monitoring of pathogen spectrum and antibiotic susceptibility patterns is paramount in an era of rapidly developing antibiotic resistance. A 2011 study evaluating the epidemiology of community-acquired UTIs in women presenting to one public and four private primary healthcare centres in Gauteng reported high rates of pathogen susceptibility to fosfomycin (95.5\%) and nitrofurantoin (91\%) and lower rates of susceptibility to amoxicillin-clavulanate (82.8\%) and co-trimoxazole (44.3\%). ${ }^{5}$ A multicentre KwaZuluNatal (KZN) obstetric department study found a decline in susceptibility to commonly used beta-lactam antibiotics between 2015 and 2019 amongst urinary Escherichia coli isolates. ${ }^{6}$

Antibiotic choices for the empiric treatment of UTIs in pregnancy must take into consideration both the efficacy and safety of the antimicrobial agents, with oral formulations preferred for outpatient treatment of UTIs. An additional real-world factor influencing empiric choices is the availability of specific drugs at individual healthcare centres. The South African Essential Drug List (EDL) currently recommends the use of empiric nitrofurantoin $100 \mathrm{mg} 6$ hourly for 5 days or a single $3 \mathrm{~g}$ dose of fosfomycin for treatment of cystitis in pregnancy. For pyelonephritis, empiric therapy with intravenous ceftriaxone is recommended. ${ }^{7}$ Nitrofurantoin and fosfomycin cannot be used for the treatment of pyelonephritis.

The worldwide problem of antimicrobial resistance (AMR) has led to the World Health Organization's publishing of the priority pathogens list, as well as the global action plan on AMR. Improving the understanding of AMR, strengthening knowledge through surveillance and optimising the use of antimicrobials are some of the key objectives of these initiatives. ${ }^{8,9}$ The obstetric population is a vulnerable patient population. Severe infections and inadequately treated infections can result in poor outcomes not only to the mother but also to the unborn infant. Application of these principles to this patient population is highly relevant.

The objective of the study was to analyse the prevalence and antimicrobial susceptibility profiles of uropathogens in the obstetric patient population at the Charlotte Maxeke Johannesburg Academic Hospital (CMJAH) over 6 years, with the aim of providing guidance on empiric therapy and recommendations for ongoing surveillance.

\section{Methods}

\section{Study setting and patient population}

The CMJAH is a 1088 bed tertiary facility located in Johannesburg, South Africa. The hospital provides numerous specialist services. This includes the obstetric department, which provides antenatal care services to 'walk-in' patients and high-risk patients (for whom basic antenatal care is inadequate) referred from the surrounding healthcare centres. The obstetric department has a 28-bedded high-risk antenatal ward and a five-bedded high-care unit. Antenatal services are delivered from Monday to Friday, whilst emergency obstetric services are available $24 \mathrm{~h}$ in the maternity admissions ward located near the main casualty of the hospital. The yearly average of antenatal clinic (ANC) visits and deliveries in the department is 21000 and 8850, respectively.

\section{Study design}

We performed a retrospective laboratory analysis of positive urine culture isolates from three areas of the CMJAH obstetric department: the antenatal clinic, maternity admissions and labour ward from 1 January 2015 to 31 December 2020.

\section{Investigation of urinary tract infection in pregnancy}

Midstream urine (MSU) samples are submitted for cell count, culture and susceptibility testing for patients with UTI symptoms and when urine dipsticks, which are routinely performed at each ANC visit, detect the presence of leucocyte esterase or nitrites. For maternity admissions and labour ward, MSU or samples from catheters in catheterised patients are submitted when the urine dipsticks show leucocyte esterase or nitrites or a septic-work-up is performed. In labour ward, samples are also submitted from patients with significant proteinuria.

\section{Laboratory testing}

Samples were transported to the on-site CMJAH National Health Laboratory Services (NHLS) Microbiology laboratory, which operates 24-h a day. Samples were processed using standard microbiological techniques. Organism identification and antimicrobial susceptibility testing (AST) were performed on automated systems (Vitek ${ }^{\circledR} 2$ and Vitek ${ }^{\circledR}$ MS, bioMerieux, France) and with manual biochemical and Kirby Bauer disk diffusion methods. Disk diffusion was used for fosfomycin susceptibility testing of E. coli isolates. During the study period, fosfomycin and nitrofurantoin testing were not performed on Enterococcus faecalis isolates as these antimicrobials are not present on the Vitek® AST panel used for Gram-positive organisms.

\section{Data collection}

Data for positive urine cultures submitted from CMJAH for the study period were extracted from the NHLS laboratory information system, TrakCare (InterSystems, USA).

\section{Data analysis}

The data extract was filtered to exclude cultures from areas other than the three relevant obstetric ones. Data were deduplicated to include only the first isolate per species per 
patient. Non-speciated coagulase-negative staphylococci (CoNS), non-saprophyticus species of CoNS, other bacterial contaminants and Candida species were removed from the data set. ${ }^{10}$

Interpretation of AST results was performed using the Clinical and Laboratory Standards Institute (CLSI) M100 guideline. ${ }^{11}$ For all antibiotics (including the beta-lactams), intermediately susceptible results were reported as resistant. Antimicrobial susceptibility testing results were reported when 30 or more isolates per species were present for that period. The CLSI analysis and presentation of cumulative AST test data M39-A4 guideline was used. ${ }^{12}$

\section{Definitions}

Extended spectrum beta-lactamase (ESBL): Non-susceptibility to one or more third-generation cephalosporins was used as a marker of ESBL production.

Carbapenem-resistant Enterobacterales (CRE): Nonsusceptibility to one or more carbapenems was used as a marker of carbapenem resistance.

\section{Statistical analysis}

Data analysis was performed using Microsoft Excel and EpiTools epidemiological calculators.

Categorical data were reported as proportions and percentages with $95 \%$ confidence interval (CI). Where AST results were not available for a specific pathogen-antibiotic combination, the denominators for percentage susceptibility calculations were adjusted to reflect this. The Z-proportions test was used to analyse differences between pathogen prevalence and susceptibility rates. Two-tailed $p$-values were reported with values $<0.05$ considered statistically significant.

\section{Ethical considerations}

An ethics waiver was obtained from the University of the Witwatersrand Human Research Ethics Committee, waiver number: W-CBP-210122-01.

\section{Results}

Following removal of common commensals, the total number of deduplicated positive culture results was 4739, with a yearly breakdown of 344 (2015), 517 (2016), 755 (2017), 998 (2018), 1151 (2019) and 974 (2020). Figure 1 illustrates the top six pathogens per year. Escherichia coli was predominant, followed by E. faecalis across all time periods. In 2020, these two organisms were the causative pathogens in $62.8 \%$ (95\% CI [59.8-65.8]) of the UTIs. The top three pathogens (including Klebsiella pneumoniae) accounted for $71.7 \%$ (95\% CI [68.9-74.5]) of all infections in 2020. Streptococcus agalactiae, Proteus mirabilis and Staphylococcus aureus were responsible for most of the remaining infections. Less commonly isolated species included Enterobacter spp.,
Citrobacter spp. and Staphylococcus saprophyticus. The prevalence of E. coli declined significantly over the study period (2015: 53.7\%, 95\% CI [48.5-58.9] and 2020: 39.7\%, 95\% CI [36.7-42.7], $p<0.0001)$.

The susceptibility results for the common Gram-negative pathogens are summarized in Table 1. Susceptibility to ampicillin was low amongst these Gram negatives. Similarly, E. coli exhibited low susceptibility rates to cotrimoxazole. Amoxicillin-clavulanate and cefuroxime showed similar rates of activity against the respective Gram-negative pathogens. The pooled 2019/2020 E. coli amoxicillin-clavulanate data demonstrated a susceptibility rate of $82 \%$ (95\% CI [79.1-84.2]). The susceptibility of E. coli to cefuroxime declined from 95\% (95\% CI [90.7-95.2]) in 2015 to $81 \%$ (95\% CI [76.4-84.5], $p<0.0001)$ in 2020. The E. coli ESBL rate increased from 5\% (95\% CI [2.5-9.1]) in 2015 to $10 \%$ (95\% CI [7.2-13.2], $p=0.04$ ) in 2020. Both nitrofurantoin and fosfomycin retained high rates of activity against E. coli. E. coli susceptibility to these two agents remained stable over the study period (nitrofurantoin 2015: 95\%, 95\% CI [90.3-97.4] vs. 2020: 93\%, 95\% CI [90.3-95.5], $p=0.359$ and fosfomycin 2015: 97\%,95\% CI [90.1-99.3] vs. 2020: $96 \%, 95 \%$ CI [93.8-98.1], $p=0.552)$. Of the 38 ESBLproducing E. coli isolates in 2020, 92\% (95\% CI [78.5-98]), 94\% (95\% CI [81.8-99.5]) and 47\% (95\% CI [32.5-62.7]) were susceptible to nitrofurantoin, fosfomycin and amoxicillinclavulanate, respectively. The emergence of carbapenemresistant K. pneumoniae in this patient population occurred in $2019(3 / 84,4 \%, 95 \%$ CI [0.8-10.6]). Nitrofurantoin susceptibility rates in K. pneumoniae were consistently low $(38 \%-46 \%)$.

All E. faecalis (except for $1 / 90$ isolates in 2016) and S. agalactiae isolates were susceptible to ampicillin. S. aureus susceptibility to cloxacillin in 2019 and 2020 was 78\% (95\%

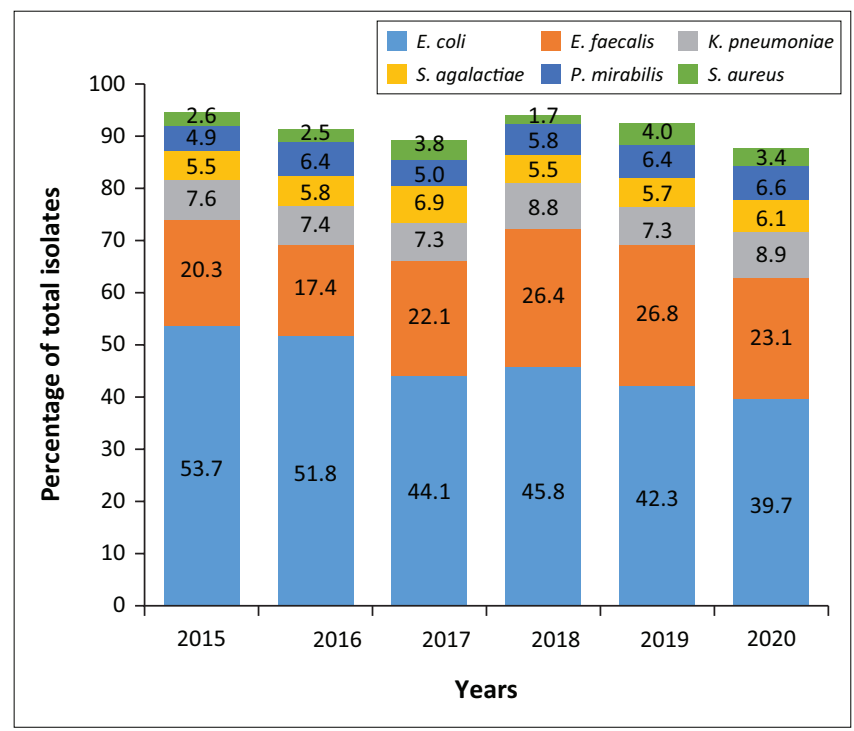

E. coli, Escherichia coli; E. faecalis, Enterococcus faecalis; K. pneumoniae, Klebsiella pneumoniae; S. agalactiae, Streptococcus agalactiae; P. mirabilis, Proteus mirabilis; S. aureus, Staphylococcus aureus.

FIGURE 1: Yearly distribution of top six uropathogens. 


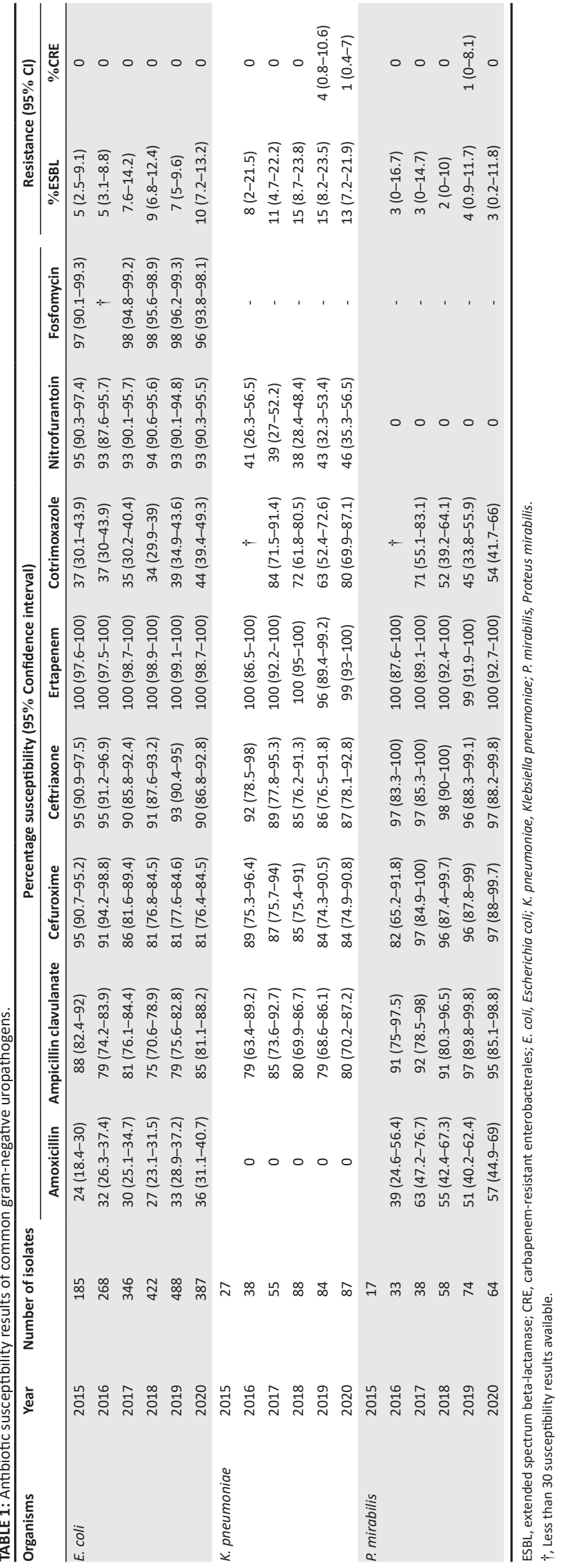




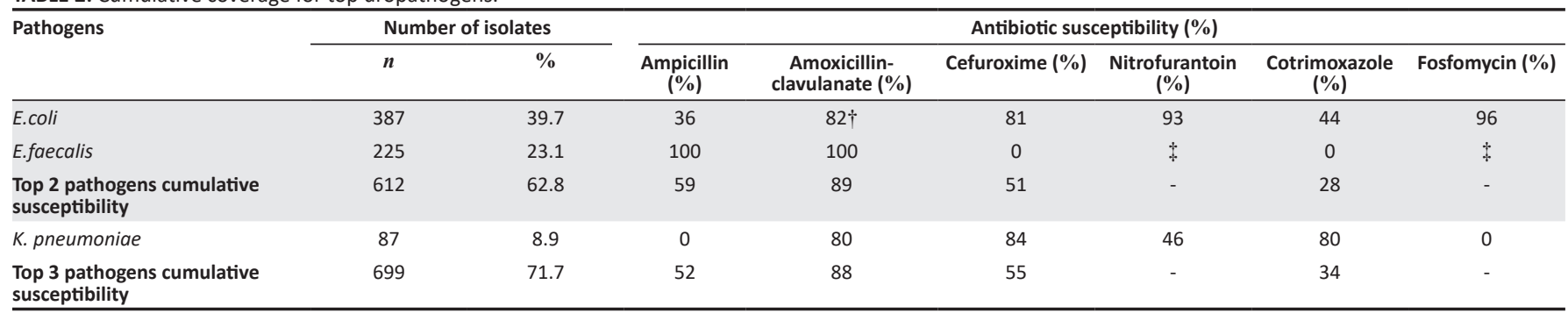

E. coli, Escherichia coli; E. faecalis, Enterococcus faecalis; K. pneumoniae, Klebsiella pneumoniae.

$\dagger$, Average of pooled 2019/2020 E. coli amoxicillin-clavulanate data.

$\ddagger$, Susceptibility data not available.

CI [64.2-87.9]) and 85\% (95\% CI [68.6-93.8]), respectively, $(p>0.05)$.

In 2020, the cumulative coverage provided by amoxicillinclavulanate for the top two and three pathogens, which comprised $62.8 \%$ and $71.7 \%$ of total isolates, was $89 \%$ (95\% CI [86.5-91.5]) (Table 2) and 88\% (95\% CI [85.6-90.4]), respectively. Cumulative coverage rates provided by nitrofurantoin and fosfomycin were not determined because of the lack of data for E. faecalis.

\section{Discussion}

The top three pathogens (in descending order: E. coli, E. faecalis and K. pneumoniae) remained consistent from 2015 to 2020. Whilst the high E. coli susceptibility rates to nitrofurantoin and fosfomycin remained stable, a significant increase in ESBLs was seen. Similarly, the ESBL rate and emergence of carbapenem resistance in the K. pneumoniae isolates is problematic.

There is a lack of national surveillance at the antenatal care and primary healthcare-level in general, where empiric therapy for UTIs is frequently prescribed. However, contemporary single centre and regional studies within South Africa can provide useful data to validate current recommendations, to signal when these recommendations need to be modified and to identify gaps in epidemiological data that need to be addressed. Regional or local changes in pathogen spectrum and proportions and their associated antibiotic susceptibility patterns may also mandate changes in recommendations in local guidelines. When resistance rate to a specific antibiotic exceeds $20 \%$, this antibiotic is no longer suitable for use as an empiric therapy choice. ${ }^{13}$ Beta-lactam antibiotics are renally excreted and reach high urinary concentrations. The European Committee of Antimicrobial Susceptibility Testing (EUCAST) and CLSI antibiotic interpretive breakpoints reflect this with specific (higher) breakpoints for uncomplicated UTIs and comments for the intermediately susceptible breakpoints, respectively. Laboratories must report AST results for UTIs accordingly.

The common pathogens identified in this study are in general consistent with the findings of other recently published South African studies. ${ }^{6,14,15}$ The relatively high number of E. faecalis infections (2016: 17.4\%) in this study is similar to that seen in the pooled 2015-2019 data for four Gauteng tertiary centres (including $\mathrm{CMJAH}$ ) but higher than that reported for 2011-2016 in KZN (4.2\%). The second most common pathogen in KZN was $K$. pneumoniae. The KZN study included Candida species, but this is unlikely to fully account for the differences. Limited Gauteng data from 2011 showed E. faecalis, whilst being the predominant Grampositive pathogen, comprised only $4 \%$ of all pathogens. ${ }^{5}$ The reason(s) for the higher E. faecalis and declining E. coli rates in this patient population are not well understood. The E. coli rates were lower than those in the given cited studies: $54.2 \%$ (Zwane et al.), 56\% (Bhola et al.) and 56\% (Lewis et al.). Comparison of centre-specific data (with CMJAH, Chris Hani Baragwanath Academic Hospital and Rahima Moosa Mother and Child Hospital based in Johannesburg and Steve Biko Academic Hospital in Tshwane) from the Zwane et al. publication may yield useful information regarding trends in E. coli rates in Gauteng obstetric patient populations.

The high rate of resistance to ampicillin and cotrimoxazole amongst the Enterobacterales in this study and globally precludes the use of these antibiotics for empiric therapy of UTI in the pregnant patient. ${ }^{16}$ The significant decline in E. coli susceptibility to cefuroxime coupled with the intrinsic resistance of enterococci to cephalosporins reduces the utility of empiric cefuroxime use at CMJAH and other settings with a significant burden of E. faecalis UTIs. Escherichia coli cefuroxime susceptibility in KZN hospitals was similarly low at $76 \% .{ }^{6}$ Amongst the E. coli isolates, amoxicillin-clavulanate susceptibility was similar to cefuroxime. However, amoxicillin-clavulanate is active against E. faecalis and thus provides superior cover when used empirically at CMJAH. Escherichia coli amoxicillin-clavulanate susceptibility reported by Zwane et al. and Bhola et al. (82\% and $78.4 \%$, respectively) was comparable to the $2019 / 2020$ CMJAH rate of $82 \%$.

The South African EDL recommends ceftriaxone as empiric therapy for pyelonephritis in pregnancy. ${ }^{7}$ The increase in E. coli ESBLs at CMJAH parallels increases seen in KZN hospital obstetric departments (2015: 9.8\% and 2016: 16.7\%) and urban and rural community healthcare centres $(11.5 \%) .{ }^{14,15}$ Escherichia coli ESBL rates in community-acquired UTIs are increasing globally. ${ }^{17}$ Whilst the CMJAH UTIs 
caused by K. pneumoniae were fewer than those as a result of E. coli, the K. pneumoniae ESBL rate is noteworthy. The pooled data for four Gauteng tertiary level antenatal care units for 2015-2019 showed ESBL rates ranging from 6\% to 9\% amongst the E. coli and K. pneumoniae isolates. ${ }^{14}$ A 2017 metaanalysis of ESBL colonisation and infection rates in African pregnant and peripartum women found a $22 \%$ prevalence of ESBLs in community urine and stool samples from Nigeria, Madagascar, South Africa and Cameroon. ${ }^{18}$ Ceftriaxone remains an appropriate empiric agent for pyelonephritis currently, but close monitoring of ESBL rates in the pregnant population is required.

Carbapenem-resistance amongst the Enterobacterales in hospital-acquired and healthcare-associated UTIs is well documented. ${ }^{19}$ The epidemiological data are lacking in this study to determine the definite origin/classification of CRE K. pneumoniae and P. mirabilis infections as community versus healthcare-associated. Determining the risk factors for ESBL and CRE UTIs in the pregnant population is an important area for further investigation. Prior antibiotic therapy, healthcare exposure, colonisation following travel to areas where multidrug-resistant organisms (MDROs) are endemic, recurrent UTIs, complicated UTIs and chronic medical conditions have been identified as risk factors for UTIs with MDROs in the general population. ${ }^{17,20}$

High rates of $E$. coli susceptibility to nitrofurantoin were maintained over the 6-year period. This is in keeping with local and global data.5,6,14,16 The multimodal mechanism of action of nitrofurantoin limits the emergence of acquired resistance. ${ }^{21}$ In addition, the pharmacokinetic properties of nitrofurantoin make it an ideal agent for the treatment of lower UTIs (good oral bioavailability, rapid clearance from serum and elimination largely in urine). Furthermore, nitrofurantoin has minimal impact on the gut microbiome in comparison with other antibiotics commonly used for UTIs. ${ }^{22}$ Proteus species are intrinsically resistant to nitrofurantoin.

Like nitrofurantoin, fosfomycin showed consistently high levels of activity against E. coli including the ESBL-producing isolates. There is limited published data for fosfomycin from other South African centres. The 2011 Lewis et al. Gauteng community-acquired UTI study reported $98.3 \%$ fosfomycin susceptibility amongst Gram-negative pathogens. ${ }^{5}$ Internationally fosfomycin susceptibility is also high amongst E. coli isolates despite use of this antimicrobial for many years. ${ }^{23}$ The activity of fosfomycin against Enterobacterales other than E. coli is not reliable. As such, CLSI and EUCAST currently only have breakpoints for E. coli. Advantages of oral fosfomycin include single dose administration (associated with good compliance), attainment of prolonged high urinary concentrations and minimal associated collateral damage such as dysbiosis and colonisation with MDROs. ${ }^{23} \mathrm{~A}$ recently published meta-analysis concluded that clinical outcomes of fosfomycin therapy for ASB and symptomatic UTIs in pregnancy are equivalent to that of other antibiotics (betalactams, sulphonamides, quinolones and nitrofurantoin). ${ }^{24}$
The high rates of ampicillin susceptibility amongst the E. faecalis and S. agalactiae isolates are in keeping with results of the other South African studies. ${ }^{6,14}$ Pooled data for 301 E. faecalis isolates from three other Gauteng tertiary ANC units, which serve a similar obstetric patient population to CMJAH (Chris Hani Baragwanath Academic Hospital, Rahima Moosa Mother and Child Hospital and Steve Biko Academic Hospital), showed a susceptibility rate of $99 \%$ to nitrofurantoin. ${ }^{14}$ High rates of E. faecalis susceptibility to nitrofurantoin have been reported internationally as well. ${ }^{25,26}$ Fosfomycin susceptibility testing of a subset of CMJAH 2015/2016 E. faecalis isolates showed high rates of susceptibility (98\%). ${ }^{27}$ Fosfomycin also shows good activity against E. faecalis in the published literature. ${ }^{25,28}$ Whilst E. faecalis nitrofurantoin and fosfomycin susceptibility data were not available for this study population specifically, it is expected that these antimicrobials will provide high levels of cover for $E$. faecalis isolates from CMJAH ANC patients. However, this must be confirmed by the CMJAHMicrobiology Laboratory through the implementation of routine testing of these isolates or the performance of periodic surveillance testing. Urinary tract infections caused by S. aureus comprised a small proportion of all infections. However, methicillinresistant S. aureus (MRSA) infections were documented in 2019 and 2020. Risk factors for UTIs caused by MRSA in pregnancy must be explored. Staphylococcus aureus has been reported as important uropathogen in parts of Africa. ${ }^{4}$

The EDL-recommended agents, nitrofurantoin and fosfomycin, provide good coverage for E. coli, the top pathogen at CMJAH and Gauteng tertiary ANCs for which published epidemiological data are available. Based on the high rate of $E$. faecalis nitrofurantoin susceptibility reported from other Gauteng tertiary ANC populations, the use of nitrofurantoin as empiric therapy is acceptable. The lower susceptibility rates of K. pneumoniae to nitrofurantoin (46\% in 2020) and the questionable activity of fosfomycin against this organism make these EDL recommended antibiotics less reliable for K. pneumoniae. As a result of the much lower proportion of UTIs caused by K. pneumoniae (8.9\% in 2020), the current practice of empiric nitrofurantoin use at $\mathrm{CMJAH}$ remains appropriate. Fosfomycin use at CMJAH is generally reserved as directed therapy for nitrofurantoin-resistant uropathogens. With the greater prevalence of K. pneumoniae in $\mathrm{KZN}$, investigation of the cumulative coverage provided by nitrofurantoin and fosfomycin in this setting is indicated. Amoxicillin-clavulanate shows good activity against the top three pathogens. However, amoxicillin-clavulanate use in pregnancy has been associated with a significant increase of necrotising enterocolitis in the neonate. ${ }^{29}$

Reductions in maternal and neonatal mortality through improved antenatal care and fewer prematurity-related complications in neonates are important components of the United Nations Sustainable Development Goals. ${ }^{30}$ Neonatal outcomes are closely related to maternal health status. Data from a Tanzanian neonatal unit demonstrated that maternal colonisation with ESBL Enterobacterales was a risk factor for neonatal colonisation and sepsis with these organisms. ${ }^{31} \mathrm{~A}$ 
German study in two neonatal units, using strain typing, showed that maternal colonising ESBL strains were related to those colonising their neonates. ${ }^{32}$ Efforts to optimise antimicrobial use (particularly in the context of UTIs as these are the most common infection type in this population) and reduce $A M R$ in the obstetric patient population will positively impact neonatal outcomes too.

Data from other provinces and different levels of care, particularly primary care (although the expectation is that lower levels of resistance will be seen at this level compared to ANCs at tertiary hospitals), are required to determine the ongoing applicability of national recommendations.

Strengths of this study include the availability of recent and longitudinal data for 6 years and the E. coli fosfomycin data (not available from other recent South African publications). This study has a number of limitations. It is a single tertiary centre study, and the epidemiology will not be representative of that in other provinces and levels of care. The CMJAH ANC sees both walk-in patients from the community and patients referred from surrounding centres. It was not possible to separate out hospitalacquired infections from community-acquired ones. It is expected that the resistance rates found in this study setting are higher than those in primary healthcare settings. Non-speciation of 47 CoNS isolates over the 6 years may have resulted in an underestimation of S. saprophyticus prevalence, but these species would not constitute more than $3 \%$ of all isolates. Cefazolin susceptibility data for the Enterobacterales was not available. However, based on the cefuroxime data, cefazolin would not be an appropriate empiric agent. Nitrofurantoin and fosfomycin data for E. faecalis were not available. However, data from other Gauteng studies show favourable susceptibility rates to these antibiotics. For the beta-lactam antibiotics categorisation of intermediate susceptibility results as 'resistant' for data analysis purposes, may have resulted in underestimation of the potential activity of these antibiotics.

Consistent with other reports from the country, our study reiterates the rising rates of resistance amongst uropathogens causing UTIs in pregnant women. Ongoing surveillance and further clinical studies in this setting are necessary to determine risk factors, effect of various antimicrobial agents and outcomes for both mother and infant. We have highlighted the limited treatment agents available. Further pharmaceutical interest in development of antimicrobial agents, which are safe during pregnancy, is both imperative and urgent because these rising resistance rates could potentially threaten the progress of the UN sustainable development goals.

\section{Acknowledgements Competing interests}

The authors declare that they have no financial or personal relationships that may have inappropriately influenced them in writing this article.

\section{Authors' contributions}

T.N., S.B. and V.C. contributed to the design and implementation of the research, to the analysis of the results and to the writing of the manuscript.

\section{Funding information}

This research received no specific grant from any funding agency in the public, commercial or not-for-profit sectors.

\section{Data availability}

The data that support the findings of this study are available from the corresponding author, T.N., upon reasonable request.

\section{Disclaimer}

The views and opinions expressed in this article are those of the authors and do not necessarily reflect the official policy or position of any affiliated agency of the authors.

\section{References}

1. Kalinderi $K$, Delkos $D$, Kalinderis $M$, Athanasiadis $A$, Kalogiannidis I. Urinary tract infection during pregnancy: Current concepts on a common multifaceted problem. J Obstet Gynaecol. 2018;38(4):448-453. https://doi.org/10.1080/01443615.2017. 1370579

2. Nicolle LE, Gupta K, Bradley SF, et al. Clinical practice guideline for the management of asymptomatic bacteriuria: 2019 update by the infectious diseases society of America. Clin Infect Dis. 2019;68(10):1611-1615. https://doi.org/10.1093/cid/ciz021

3. World Health Organization, editor. WHO recommendations on antenatal care for a positive pregnancy experience [homepage on the Internet]. Geneva: World Health Organization; 2016 [cited 2021 Aug 10]. Available from: https://apps.who. int/iris/bitstream/handle/10665/250796/9789241549912-eng.pdf

4. Gilbert NM, O'Brien VP, Hultgren S, Macones G, Lewis WG, Lewis AL. Urinary tract infection as a preventable cause of pregnancy complications: Opportunities, challenges, and a global call to action. Glob Adv Health Med. 2013;2(5):59-69. https://doi.org/10.7453/gahmj.2013.061

5. Lewis DA, Gumede LYE, Van der Hoven LA, et al. Antimicrobial susceptibility of organisms causing community-acquired urinary tract infections in Gauteng province, South Africa. S Afr Med J. 2013;103(6):377. https://doi.org/10.7196/SAMJ.6722

6. Bhola P, Mvelase NR, Balakrishna Y, Mlisana KP, Swe Swe-Han K. Antimicrobial susceptibility patterns of uropathogens isolated from pregnant women in KwaZulu-Natal Province: 2011-2016. S Afr Med J. 2020;110(9):872. https://doi. org/10.7196/SAMJ.2020.v110i9.14468

7. Department of Health Republic of South Africa. Standard treatment guidelines and essential medicines list for South Africa [homepage on the Internet]. Primary Healthcare Level; 2020 [cited 2021 Aug 10]. Available from: http://www. kznhealth.gov.za/pharmacy/PHC-STG-2020.pdf

8. World Health Organization. WHO priority pathogens list for R\&D of new antibiotics [homepage on the Internet]. 2017 [cited 2020 Jun 17]. Available from: www.who. int/bulletin/volumes/94/9/16-020916.pdf

9. World Health Organization. Global action plan on antimicrobial resistance [homepage on the Internet]. 2015 [cited 2021 Aug 10]. Available from: http:// www.emro.who.int/health-topics/drug-resistance/global-action-plan.html

10. Centres for Disease Control and Prevention. National healthcare safety network (NHSN) patient safety component manual [homepage on the Internet]. 2021 [cited 2021 Aug 10]. Available from: https://www.cdc.gov/nhsn/pdfs/pscmanual/ pcsmanual_current.pdf

11. Clinical and Laboratory Standards Institute. Performance standards for antimicrobial susceptibility testing. 30th ed. CLSI guideline M100. Wayne, PA: CLSI; 2020.

12. Clinical and Laboratory Standards Institute. Analysis and presentation of cumulative antimicrobial susceptibility test data. 4th ed. CLSI guideline M39-A4. Wayne PA: CLSI; 2014.

13. Gupta K, Hooton TM, Naber KG, et al. International clinical practice guidelines for the treatment of acute uncomplicated cystitis and pyelonephritis in women: A 2010 update by the infectious diseases society of America and the European Society for microbiology and infectious diseases. Clin Infect Dis.
2011;52(5):e103-e120. https://doi.org/10.1093/cid/ciq257

14. Zwane T, Shuping L, Perovic O. Etiology and antimicrobial susceptibility of pathogens associated with urinary tract infections among women attending antenatal care in four South African tertiary-level facilities, 2015-2019. Antibiotics. 2021;10(6):669. https://doi.org/10.3390/antibiotics10060669

15. Kubone PZ, Mlisana KP, Govinden U, Abia ALK, Essack SY. Antibiotic susceptibility and molecular characterization of uropathogenic Escherichia coli associated with community-acquired urinary tract infections in urban and rural settings in South Africa. Trop Med Infect Dis. 2020;5(4):176. https://doi.org/10.3390/tropicalmed5040176 
16. Kot B. Antibiotic resistance among uropathogenic Escherichia coli. Pol J Microbiol. 2019;68(4):403-415. https://doi.org/10.33073/pjm-2019-048

17. Lee DS, Lee S-J, Choe H-S. Community-acquired urinary tract infection by Escherichia coli in the era of antibiotic resistance. BioMed Res Int. 2018;2018:7656752. https://doi.org/10.1155/2018/7656752

18. Bulabula ANH, Dramowski A, Mehtar S. Maternal colonization or infection with extended-spectrum beta-lactamase-producing Enterobacteriaceae in Africa: A systematic review and meta-analysis. Int J Infect Dis. 2017;64:58-66. https://doi. org/10.1016/j.ijid.2017.08.015

19. Zowawi HM, Harris PNA, Roberts MJ, et al. The emerging threat of multidrug resistant Gram-negative bacteria in urology. Nat Rev Urol. 2015;12(10):570-584. https://doi.org/10.1038/nrurol.2015.199

20. Bader MS, Loeb M, Leto D, Brooks AA. Treatment of urinary tract infections in the era of antimicrobial resistance and new antimicrobial agents. Postgrad Med. 2020;132(3):234-250. https://doi.org/10.1080/00325481.2019.1680052

21. Howard B. Nitrofurantoin. In: xPharm: The comprehensive pharmacology reference [homepage on the Internet]. Elsevier; 2007 [cited 2021 Jul 6]; p. 1-6. Available from: https://linkinghub.elsevier.com/retrieve/pii/B9780080552323622901

22. Mavromanolakis E, Maraki S, Samonis G, Tselentis Y, Cranidis A. Effect of norfloxacin, trimethoprim-sulfamethoxazole and nitrofurantoin on fecal flora of women with recurrent urinary tract infections. J Chemother. 1997;9(3):203-207. https://doi.org/10.1179/joc.1997.9.3.203

23. Kranz J, Helbig S, Mandraka F, Schmidt S, Naber KG. The revival of old antibiotics for treatment of uncomplicated urinary tract infections in the era of antibiotic stewardship. Curr Opin Urol. 2017;27(2):127-132. https://doi.org/10.1097/ MOU.0000000000000365

24. Wang T, Wu G, Wang J, et al. Comparison of single-dose fosfomycin tromethamine and other antibiotics for lower uncomplicated urinary tract infection in women and asymptomatic bacteriuria in pregnant women: A systematic review and metaanalysis. Int J Antimicrob Agents. 2020;56(1):106018. https://doi.org/10.1016/j. ijantimicag.2020.106018
25. Keepers TR, Gomez M, Celeri C, Krause KM, Biek D, Critchley I. Fosfomycin and comparator activity against select Enterobacteriaceae, Pseudomonas, and Enterococcus urinary tract infection isolates from the United States in 2012 Infect Dis Ther. 2017;6(2):233-243. https://doi.org/10.1007/s40121-0170150-5

26. Ghalavand Z, Alebouyeh M, Ghanati K, Azimi L, Rashidan M. Genetic relatedness of the Enterococcus faecalis isolates in stool and urine samples of patients with community-acquired urinary tract infection. Gut Pathog. 2020;12:42. https://doi. org/10.1186/s13099-020-00380-7

27. Mothibi LM, Bosman NN, Nana T. Fosfomycin susceptibility of uropathogens at Charlotte Maxeke Johannesburg Academic Hospital. S Afr J Infect Dis. 2020;35(1):a173. https://doi.org/10.4102/sajid.v35i1.173

28. Das A, Banerjee T, Anupurba S. Susceptibility of nitrofurantoin and fosfomycin against outpatient urinary isolates of multidrug-resistant Enterococci over a period of 10 years from India. Microb Drug Resist. 2020;26(12):1509-1515. https://doi.org/10.1089/mdr.2019.0044

29. Kenyon S, Taylor D, Tarnow-Mordi W, ORACLE Collaborative Group. ORACLEantibiotics for preterm prelabour rupture of the membranes: Short-term and long-term outcomes. Acta Paediatr. 2007;91:12-15. https://doi.org/10.1111/j. 1651-2227.2002.tb00153.x

30. United Nations. Sustainable development goals [homepage on the Internet]. 2015 [cited 2021 Aug 10]. Available from: https://sdgs.un.org/2030agenda

31. Marando R, Seni J, Mirambo MM, et al. Predictors of the extended-spectrum-beta lactamases producing Enterobacteriaceae neonatal sepsis at a tertiary hospital Tanzania. Int J Med Microbiol. 2018;308(7):803-811. https://doi.org/10.1016/j. ijmm.2018.06.012

32. Denkel LA, Schwab F, Kola A, et al. The mother as most important risk factor for colonization of very low birth weight (VLBW) infants with extended-spectrumlactamase-producing Enterobacteriaceae (ESBL-E). J Antimicrob Chemother. 2014 Aug 1;69(8):2230-7. https://doi.org/10.1093/jac/dku097 